\title{
Estudio comparado: formación para la ciudadanía en el primer ciclo de Educación Secundaria en Uruguay y Cataluña
}

\section{Comparative study: training for citizenship in the first cycle of Secondary Education in Uruguay and Cataluña}

\begin{abstract}
ISSN 1510-2432 - ISSN 1688-9304 (en línea) - DOI: https://doi.org/10.18861/cied.2018.9.2.2854
Juan Manuel Sarochar

Doctor en Educación, Universidad Autónoma de Barcelona (España). Magíster en Política y Gestión de la Educación, Centro Latinoamericano de Economía Humana (Uruguay). Diploma en Pedagogía para la Formación de Jóvenes y Adultos, Centro de Cooperación Regional para la Educación de Adultos en América Latina y el Caribe (México). Docente, Universidad de la Empresa (Uruguay). Investigador en ciudadanía y educación, y en procesos migratorios y educación.
\end{abstract}

Fecha de recibido: 07/08/2018

Fecha de aceptado: 23/09/2018

\section{Resumen}

El presente artículo expone parte del contenido de una Tesis Doctoral realizada entre fines de 2014 y principios de 2018. El tema contextual del estudio es la formación para la ciudadanía en el ámbito de la educación formal. El objetivo general de la investigación consiste en comparar Uruguay con Cataluña en lo que refiere a los procesos de formación para la ciudadanía, a nivel curricular y normativo, en el primer ciclo de Enseñanza Secundaria. El estudio se realizó mediante la aplicación de la metodología de investigación comparada, desde un enfoque de análisis de tipo cualitativo. Las fuentes de información a las que se recurrió fueron documentos, específicamente textos curriculares y normativos vigentes a la fecha del estudio en ambos territorios, así como informantes calificados. Las técnicas aplicadas para la recolección de información fueron el análisis documental y la entrevista. Los resultados obtenidos muestran que las dinámicas de formación para la ciudadanía que tienen lugar en un territorio y en otro acusan marcadas diferencias.

Palabras clave: estudio comparado, educación para la ciudadanía, educación secundaria

\begin{abstract}
This article presents part of the content of a Doctoral Thesis carried out between the end of 2014 and the beginning of 2018. The contextual subject of the study is education for citizenship in the field of formal education. The overall aim of the research is to compare Uruguay with Catalonia in terms of education processes for citizenship, at the curricular and normative level, in the first cycle of Secondary Education. The study was carried out through the application of the methodology of comparative research, from a qualitative analysis approach. Documents, specifically curricular and normative texts in force at the date of the study in both territories were used as the main sources of information. Interviews to qualified informants were also resorted to. In accordance to the above, the techniques applied for the collection of information were the documentary analysis and interviews. The results obtained show that the dynamics of education for citizenship in either territory are clearly different.
\end{abstract}

Keywords: comparative study, education for citizenship, secondary education 


\section{Marco conceptual y planteamiento del tema. Problema y pregunta de investigación}

A partir de las últimas décadas del siglo veinte, especialmente desde mediados de los años ochenta e inicio de los noventa, cuando en Latinoamérica finalizaban los regímenes dictatoriales que tuvieron lugar en la región y en Europa Occidental comenzaba a concretarse lo que hoy se conoce como el proyecto de la Unión Europea, se incrementó notoriamente el interés tanto político como social por la cuestión de la formación para la ciudadanía en el ámbito escolar (Audigier, 2000; Meheut, 2000; Pedró, 2003; Cox, 2006; Buxarrais, 2009; Redon, 2010; López de Cordero, 2011). Dicho interés se evidencia, por ejemplo, en la intensa convocatoria efectuada desde diversas instancias organizacionales de la Unión Europea para que los países miembros aborden esta cuestión en sus respectivos sistemas educativos. Asimismo, en el contexto de integración del MERCOSUR (Mercado Común del Sur), en el cual se inscribe Uruguay, han surgido diversas recomendaciones para que los países integrantes del bloque trabajen en el desarrollo de planes y programas que tengan por objetivo profundizar (cualitativa y cuantitativamente) en los procesos educativos vinculados a la formación para la ciudadanía tanto en el ámbito de la Educación Primaria como en el de la Educación Secundaria.

Así, en ambos escenarios de integración regional ha adquirido un importante impulso la idea de que la formación para la ciudadanía es indispensable para lograr el desarrollo democrático y armonioso de las sociedades actuales, en cuanto favorecedora de la cohesión social, la comprensión mutua y la solidaridad, el diálogo intercultural e interreligioso, a la vez que contribuye a promover el principio de igualdad entre los seres humanos (Pedró, 2003; Pagès, 2009; Aiello de Almeida, 2010; López de Cordero, 2010).

En este sentido se entiende que los espacios de educación formal, aunque no exclusivamente, desempeñan un papel fundamental en la promoción de la formación para la ciudadanía, y no solo por el hecho de que existan asignaturas cuyo centro sea, justamente, coadyuvar a ello, sino porque se asume que los centros educativos son, en sí mismos, espacios de vivencia de variadas interacciones sociales en las que tanto niños como jóvenes tienen la oportunidad de experimentar y aprehender algunos de los valores y principios ciudadanos más fundamentales, imprescindibles para el pleno ejercicio de la ciudadanía política y social (Buxarrais, 2009; Redon, 2010).

De tal modo, se concibe que los centros educativos son sitios donde (en relación -discordante o no- con las dinámicas del entorno familiar, del grupo de pares, de otros grupos sociales y los medios de comunicación) las nuevas generaciones dan sus primeros pasos en aspectos básicos del proceso de socialización y de convivencia ciudadana.

Por una parte, no sorprende que la cuestión de la formación para la ciudadanía se haya transformado rápidamente, en muchas regiones del mundo, en una de las prioridades de las agendas educativas actuales, esto es, de las reformas y políticas educativas a la interna de los sistemas educativos nacionales así como también en los procesos de armonización educativa entre estados (Pedró, 2003; González, 2011; Red Eurydice, 2012).

Por otra parte, el abanico de estrategias y acciones impulsadas para el fomento de la formación para la ciudadanía se ha visto hondamente trastocado en las últimas décadas a causa de la incidencia de diversas y complejas dinámicas de alcance mundial: globalización económica, tecnológica y cultural, intensificación de los procesos migratorios de escala regional e intercontinental, transformaciones en el ámbito del poder político de los estados, 
surgimiento de nuevos y muy influyentes actores en el escenario político internacional, nuevas formas de gobernanza local y global y aparición de nuevas formas de interacción y de acción social, por citar solo algunas de las más generales. Dinámicas éstas que también han afectado profundamente la misma noción de ciudadanía (Berger y Huntington, 2002; Held y McGrew, 2003).

En este sentido no son pocos los autores que utilizan los términos de confusión, indefinición, desfiguración, tensión o distorsión para referirse a la crisis por la que atraviesa en la actualidad la noción de ciudadanía (Fariñas, 1999; Salazar, 2001; Pérez, 2002; Held y McGrew, 2003; Pedró, 2003; Aláez, 2005; Andrés, 2007; Horrach, 2009; Anchustegui, 2011). De tal modo, no caben dudas de que si su abordaje teórico por parte de los integrantes de la academia se ha vuelto tan complejo y discutido, no son menos extensos ni profundos los debates establecidos en torno al abordaje e instrumentalización de la cuestión ciudadana a la interna de los sistemas educativos, en las instituciones escolares, en las aulas.

En este sentido, el impetuoso interés por incluir formación para la ciudadanía en las instituciones escolares ha promovido el surgimiento de importantes discusiones en torno a preguntas como: ¿Qué noción o nociones de ciudadanía transmite la escuela contemporánea?, ¿Con qué intereses explícitos?, ¿Con qué intereses implícitos?, ¿Cómo lo hace?, ¿Efectivamente lo hace?, ¿Qué dimensiones de la ciudadanía se ven más fomentadas en la escuela?, ¿Qué dimensiones de la ciudadanía se ven más relegadas en la escuela?, ¿Qué visión -explícita e implícita- de hombre resguarda la formación para la ciudadanía en el ámbito escolar?

Tal como lo evidencian estas preguntas, los procesos de formación para la ciudadanía no representan una cuestión trivial, desprovista de cargas implícitas o subterráneas (Buxarrais, 2009; Pagès, 2009).

Entonces, es debido al interés generado por la importancia y profundidad que alcanzan en la actualidad estos debates e interrogantes que nuestro tema general de investigación es la formación para la ciudadanía en el ámbito de la educación formal. En este marco temático nuestro problema de investigación son las dinámicas de los procesos de formación para la ciudadanía, a nivel curricular y normativo, en el primer ciclo de Enseñanza Secundaria en Cataluña y Uruguay. Del mismo deriva la siguiente pregunta de investigación: ¿En cuáles configuraciones se asemejan y en cuáles se diferencian los procesos de formación para la ciudadanía, a nivel curricular y normativo, en el primer ciclo de Enseñanza Secundaria en Cataluña y en Uruguay?

Las razones por las que se decidió comparar los casos de Uruguay y Cataluña se ajustan a lo que en la literatura académica sobre investigación se denomina como selección por motivos intrínsecos. Stake (1998) y Álvarez y San Fabián (2012) plantean que este tipo de selección tiene lugar cuando los casos considerados guardan un valor en sí mismos para el investigador o, dicho de otro modo, el investigador tiene un interés intrínseco en los mismos.

Concretamente, la elección de Uruguay responde a que es nuestro país de residencia permanente, por lo cual tenemos especial interés en conocer cómo se desarrolla aquí la dinámica investigada. Por su parte, la selección de Cataluña obedece a que es donde se sitúa el centro universitario de filiación de nuestro proceso de investigación doctoral, lo cual desde un principio despertó el interés de que fuera el escenario a comparar con Uruguay. 
Ahora bien, cabe explicitar que como soporte empírico para la elección de ambos escenarios se tuvo en cuenta la existencia de aspectos cualitativos de orden histórico, cultural, jurídico, educativo y demográfico que acercan y, por ello, hacen viable el ejercicio de comparación entre Uruguay y Cataluña.

\section{Metodología}

El perfil de este estudio hace que el mismo se encuadre dentro de dos perspectivas o líneas generales de investigación: la investigación comparada y la investigación cualitativa.

Una de las fuentes de información utilizadas es la fuente documental. En este sentido se analizaron comparativamente 35 textos curriculares de asignaturas correspondientes al primer ciclo de Educación Secundaria en Uruguay, del Plan Reformulación 2006 así como 43 textos curriculares de asignaturas pertenecientes al primer ciclo de Educación Secundaria en Cataluña establecidos por el Decreto 143/2007. El criterio general aplicado para la selección de los textos curriculares fue cubrir todas las áreas de conocimiento de este ciclo educativo en ambos territorios.

Asimismo se analizaron comparativamente textos normativos. En el caso de Uruguay se consideró la Ley General de Educación (Ley N ${ }^{0}$ 18.437) y el Estatuto del Estudiante de Educación Media (Acta N ${ }^{\circ} 47$ del Consejo Directivo Central; Resolución Nº 2 del 8 de julio de 2005). En el caso de Cataluña se consideraron la Ley de Educación de Cataluña (Ley 12/2009) y el Decreto 143/2007 por el que se establece la ordenación de las enseñanzas de la Educación Secundaria Obligatoria. Para el análisis se consideraron las leyes de educación por cuanto representan, en Uruguay y Cataluña, el marco normativo más general que regula el Ciclo Básico y la Educación Secundaria Obligatoria, respectivamente. Por su parte, el Estatuto del Estudiante de Educación Media (en Uruguay) y el Decreto 143/2007 (en Cataluña) son los cuerpos normativos que regulan con especificidad el primer ciclo de Educación Secundaria en cada uno de los territorios comparados.

La otra fuente de información a la que se recurrió consistió en informantes calificados. El tipo de muestreo utilizado fue el denominado muestreo discrecional (también conocido como muestreo intencional o muestreo por juicio) el cual se utiliza en Ciencias Sociales para seleccionar una muestra de informantes siguiendo criterios cualitativos tales como la orientación brindada por expertos académicos acerca de personas específicas que es aconsejable entrevistar, la identificación de individuos que en los textos académicos figuran como especialistas o referentes en la materia que se investiga o la identificación de individuos que, según el juicio profesional del propio investigador, pueden brindar información de relevancia acerca del tema-objeto de investigación.

Tanto en el caso de Cataluña como en el de Uruguay la selección de los informantes se realizó mediante la confluencia de la orientación brindada por expertos en educación y la identificación por nuestra parte de informantes que consideramos idóneos para obtener de ellos datos relevantes acerca de nuestro tema-objeto de investigación.

En total fueron entrevistados 18 informantes calificados distribuidos según los siguientes atributos: 4 docentes ( 2 en Cataluña, 2 en Uruguay) en situación laboral de retiro que ejercieron la enseñanza en Educación Secundaria Obligatoria/Ciclo Básico en asignaturas del ámbito de Ciencias Sociales; 7 docentes (3 en Cataluña, 4 en Uruguay) en situación laboral activa con más de diez años de docencia en Educación Secundaria Obligatoria/ Ciclo Básico en asignaturas del ámbito de Ciencias Sociales y 7 docentes (3 en Cataluña, 4 en Uruguay) actualmente integrantes de equipos de dirección de centros educativos. 
Cabe señalar como aspecto especialmente relevante que más que enfatizar la cantidad de entrevistas que pudieran ser realizadas se consideró la dimensión cualitativa de las mismas. Se aplicó una entrevista de tipo semiestructurada y en profundidad, lo que hace que las explicaciones, los fundamentos, los puntos de vistas personales y hasta las indecisiones manifestadas por los entrevistados adquieran relevancia interpretativa más que la reiteración estadística de determinadas respuestas.

Asimismo cabe destacar que la aplicación de la entrevista semiestructurada y en profundidad tuvo como propósito esencial contextualizar mínimamente, desde un punto de vista interpretativo, los resultados obtenidos a través del análisis documental instrumentado.

\section{Resultados obtenidos y reflexiones en torno a los mismos}

En el caso catalán, un aspecto que claramente resalta en el texto del Decreto 143/2007, en el de la Ley de Educación de Cataluña (Ley 12/2009) y en los documentos curriculares analizados es que la cuestión de la formación para la ciudadanía se encuentra planteada, por el primero y el segundo, de forma transversal a la institución educativa, es decir, expresan que dicha formación debe estar incluida en todas las actividades desarrolladas en el marco del centro educativo. Asimismo, los tres textos lo plantean de forma transversal al aula de clases, esto es, al quehacer específico desarrollado desde cada una de las asignaturas de la Educación Secundaria Obligatoria.

Esto supone un primer aspecto muy importante a considerar en nuestro análisis: la formación para la ciudadanía es propuesta para su abordaje desde la interrelación de dos ámbitos escolares distintos pero intrínsecamente complementarios uno del otro: el institucional y el de aula.

Concretamente, acerca de esta doble transversalidad que aquí se alude, el Decreto 143/2007 expresa lo siguiente:

“Los objetivos que se establecen [...] en relación con la educación para la ciudadanía y los derechos humanos, tienen que formar parte del proyecto educativo de centro a fin de que haya continuidad entre los valores que se trabajan en las diferentes materias y la tutoría, así como en el resto de actividades que se desarrollan en el marco del centro". (Artículo 8.3)

“Cada centro elaborará su proyecto educativo, en el que, además de los valores, objetivos y prioridades de actuación, especificará los principios básicos para el desarrollo curricular y el tratamiento transversal en las diferentes materias de la Educación para la ciudadanía y los derechos humanos". (Artículo 23.3)

Al yuxtaponer el significado de estas disposiciones del Decreto 143/2007 al contenido de la Ley de Educación de Cataluña (Ley 12/2009) se encuentran dos concomitancias importantes. Por un lado, el texto de la ley también enfatiza en el rol sustantivo que debe tener el ámbito institucional en los procesos de formación para la ciudadanía. Así, su Artículo 79 expresa que los criterios de organización pedagógica que adopten los centros en las etapas de la educación básica deben contribuir, entre otras cuestiones, a: “Educar a los alumnos en la responsabilidad de ejercer la ciudadanía activa a través de la participación en los asuntos de la comunidad". 
Por otro lado, la Ley 12/2009 destaca en reiteradas ocasiones el lugar esencial que debe ocupar en cada institución escolar el proyecto educativo de centro. En este sentido el Artículo 91 señala que:

“Todos los centros vinculados al Servicio de Educación de Cataluña deben disponer de proyecto educativo. En el marco del ordenamiento jurídico, el proyecto educativo, que es la máxima expresión de la autonomía de los centros educativos, recoge la identidad del centro, explicita sus objetivos y orienta y da sentido a su actividad con la finalidad de que los alumnos alcancen las competencias básicas y el máximo aprovechamiento educativo. El proyecto educativo incorpora el carácter propio del centro".

Si bien en este caso, a diferencia de lo que ocurre en el Decreto 143/2007, no se menciona de manera explícita la centralidad que debe ocupar en el proyecto educativo de centro la formación para la ciudadanía, sí se ratifica o remarca de manera explícita la centralidad que debe tener el proyecto educativo de centro en la vida de la institución escolar. Por tanto, de forma indirecta la Ley 12/2009 brinda soporte normativo a la condición de esencialidad que en la escuela debe alcanzar la formación para la ciudadanía a través de su inscripción en el proyecto educativo, tal como lo establece el Decreto 143/2007.

Profundizando en lo específico de la transversalidad de la formación para la ciudadanía en el ámbito de aula cabe considerar que una de las ocho competencias básicas transversales que el Decreto 143/2007 define para la educación obligatoria es la denominada Competencia social y ciudadana. Según expresa el citado decreto, la finalidad central de cada una de las distintas áreas curriculares y materias es la promoción de todas las competencias básicas. Dicho de otro modo, la promoción de cada una de las competencias básicas debe lograrse como consecuencia del trabajo realizado en las distintas áreas o materias, por lo cual se insiste en que la eficacia en la consecución de las competencias básicas depende de una buena coordinación de las actividades escolares desarrolladas desde todas las áreas curriculares.

“La organización de las actividades en el aula y el funcionamiento de los centros, las actividades docentes, las formas de relación y de comunicación que se establecen entre la comunidad educativa y la relación con el entorno, contribuirán a la consolidación de las competencias básicas. Las actividades complementarias y extraescolares pueden favorecer, también, la consecución de las competencias básicas". (Decreto 143/2007; Artículo 7.4)

“Las competencias básicas se desarrollarán en las diferentes materias y con actividades de diferentes grados de complejidad que comporten conexiones entre contenidos intradisciplinarios o de la propia materia e interdisciplinarios o de las diferentes materias, y se integrarán las diferentes experiencias y aprendizajes de los alumnos". (Decreto 143/2007; Artículo 8.4)

"La finalidad central de cada una de las áreas curriculares es el desarrollo de las competencias básicas, teniendo en cuenta que cada una de las áreas o materias contribuye al desarrollo de distintas competencias y, a su vez, cada una de las competencias básicas se logrará como consecuencia del trabajo en varias áreas o materias. [...] Y para conseguirlo es clave la organización del centro y de las aulas: la articulación de los distintos ámbitos de organización del profesorado como los ciclos y los niveles; la participación del alumnado en la dinámica del centro y en el propio proceso de aprendizaje [...]". (Decreto 143/2007; Anexo 1) 
"Los objetivos y los contenidos de cada una de las áreas curriculares han de tener en cuenta el desarrollo integral de todas las competencias básicas [...]" (Decreto 143/2007; Anexo 1)

Estas disposiciones establecen, en definitiva, que la Competencia social y ciudadana (junto a las restantes) debe permear el currículo de la Educación Secundaria Obligatoria de manera transversal a las distintas áreas o materias. Es muy importante resaltar que esto está planteado así a pesar de que existen dos materias que desde diferentes perspectivas refieren directamente a la cuestión de la ciudadanía (Educación para la ciudadanía y derechos humanos y Educación ético-cívica). Dicho de otro modo, la presencia de ambas materias, que tienen especificidad curricular en lo que es la formación para la ciudadanía, no implica que las restantes materias estén planteadas de manera desvinculada de dicha formación.

Por una parte, más allá de que son muchas las expresiones del Decreto 143/2007 que refuerzan esta intención de transversalidad de la formación para la ciudadanía, es importante señalar que hemos evidenciado el hecho de que los currículos de las distintas materias de la Educación Secundaria Obligatoria efectivamente incorporan tal formación a sus respectivas especificidades a través del abordaje de los aspectos más nucleares de la Competencia social y ciudadana. Si bien esta incorporación no se produce -como es de esperar que suceda- con igual contundencia en todos los casos ${ }^{1}$, es destacable el hecho de que tal competencia realmente conste explicitada en el discurso de los distintos currículos.

Por otra parte, al analizar el texto de la Ley de Educación de Cataluña (Ley 12/2009) encontramos que en el mismo, sin que existan referencias específicas a la competencia social y ciudadana igualmente se insiste en la transversalidad que debe tener la formación para la ciudadanía en el currículo escolar. Así, el Artículo 52 de la ley expresa que el currículo debe estar orientado, entre otras finalidades, a "Capacitar a los alumnos para el ejercicio de la ciudadanía, con respeto a los derechos y libertades fundamentales de las personas y a los principios básicos de la convivencia democrática". Asimismo, el Artículo 57 establece que: "[...] los currículos de la educación básica deben orientarse a la adquisición de las competencias básicas, que deben contribuir al desarrollo personal de los alumnos y a la práctica de la ciudadanía activa [...]".

Al yuxtaponer la información obtenida a partir de las entrevistas realizadas con la observación antes manifestada acerca de que (según lo expresado por el Decreto 143/2007, la Ley de Educación de Cataluña y los textos curriculares de la Educación Secundaria Obligatoria) la formación para la ciudadanía es planteada de forma transversal al ámbito institucional y al ámbito de aula, como aspecto relevante para nuestro análisis hallamos que muchas de las percepciones brindadas por los informantes calificados consultados coinciden con tal observación.

En este sentido los informantes han proporcionado ejemplos concretos de cómo se instrumenta en la realidad de los centros educativos esta doble transversalidad identificada a nivel curricular y normativo. En referencia al fomento de la participación ciudadana activa en el alumnado un directivo de centro educativo (Barcelona, enero de 2016) expresó lo siguiente: 
“En general, se trata de fomentar la participación del alumnado en todas las esferas que conforman al centro educativo, partimos de que es participación ayudar a decidir colectivamente, es participación realizar actividades de servicio en otras instituciones, es participación colaborar en el cuidado ambiental de todos los espacios del centro, es participación la intervención mediadora en las situaciones de conflicto..."

Por su parte, al referirse también al fomento de la participación ciudadana activa en el alumnado, otro directivo entrevistado (Barcelona, enero de 2016) expresó lo siguiente:

“Este propósito educativo [el de formar para la participación ciudadana activa] no recae solamente sobre las asignaturas que, por los contenidos que desarrollan, más se vinculan a la cuestión de la participación ciudadana (Educación para la ciudadanía y los derechos humanos y Educación ético-cívica), sino que es una tarea asumida por los docentes de todas las asignaturas y en todos los espacios institucionales".

También son importantes las expresiones de un tercer directivo entrevistado (Palafrugell, Girona, julio de 2016) que, aunque breves, son contundentes respecto a la transversalidad a la que se viene aludiendo:

"Puedo decirte que los contenidos de ciudadanía son unos contenidos transversales tratados desde diferentes puntos de vista en cada una de las diferentes materias curriculares de la Educación Secundaria Obligatoria. Por supuesto que habrá algunos espacios en donde su tratamiento pueda ser más exhaustivo que en otros, por ejemplo en las áreas de sociales, tutoría, etc."

Es oportuno citar también los aportes que brindó un docente (profesor de Ciencias Sociales, Barcelona, enero de 2016) respecto al fomento de la participación estudiantil como una de las dimensiones de la formación para la ciudadanía:

“De acuerdo con las disposiciones normativas debemos buscar que la forma de integración del alumno al centro educativo, a la comunidad educativa, sea a través de la participación. Pero creo que los docentes lo hacemos no solo porque lo establecen las normas, tampoco por el simple hecho de animar cualquier tipo de participación, sino con la intención de forjar una participación socialmente responsable. Además, queda claro que entendemos la participación estudiantil en un sentido amplio, así como la participación ciudadana adulta es concebida desde múltiples aristas; debe darse en todos los espacios comunes de la institución como un proceso cotidiano, en las aulas, y fomentada desde todos los actores institucionales: dirección, profesorado, etc."

La información recabada a partir del análisis documental referente a la transversalidad que posee la formación para la ciudadanía en el ámbito de la Educación Secundaria Obligatoria así como la obtenida a partir de las entrevistas realizadas cobran especial importancia para nuestro análisis si las entendemos desde los aportes de diversos autores que recalcan la idea de que una formación para la ciudadanía de carácter integral ${ }^{2}$ no puede desarrollarse a cabalidad si no están dadas condiciones de cooperación y de transversalidad en/entre el nivel de aula y el nivel institucional en lo que respecta a la posibilidad de participación, de exposición de argumentos, de diálogo, de resolución de conflictos, de logro de acuerdos y de toma de decisiones por parte del alumnado. 
En este sentido resulta interesante observar que en las expresiones de los informantes calificados consultados, tal como lo evidencian las citas realizadas, los niveles institucional y de aula son considerados de forma integrada en el propósito de formar para la ciudadanía o, dicho de otro modo, no son percibidos como niveles disgregados o inconexos en la consecución de tal acción formativa.

En el caso uruguayo, hecho el análisis del texto de la Ley de Educación ( $N^{\circ}$ 18.437), del Estatuto del Estudiante de Educación Media (CODICEN, 2005) y de los currículos de las asignaturas del Ciclo Básico de Educación Secundaria (Reformulación 2006) se halló como aspecto relevante para este estudio el hecho de que ninguno de estos documentos manifiesta el propósito de que la formación para la ciudadanía deba ser abordada en los centros educativos de Ciclo Básico a nivel institucional, es decir, que ésta deba permear o alcanzar todas las actividades desarrolladas en el marco de cada centro educativo.

A este respecto, las entrevistas realizadas han permitido percibir algunas dimensiones concretas que alcanza esta situación detectada en el conjunto de los documentos analizados. Un directivo de centro educativo (Montevideo, junio de 2016) manifestó lo siguiente:

"A nivel de Educación Secundaria si bien hay cierta intencionalidad implícita, débilmente implícita diría, de formar para la ciudadanía desde todos los ámbitos institucionales, el hecho es que escrito hay poco o nada, los programas de asignatura se centran en lo que el docente debe enseñar específicamente sobre tal o cual asignatura. Quiero decirte que, por ejemplo, el currículo de matemáticas explicita contenidos a enseñar únicamente de matemáticas, de ahí que el profesor de esta materia quiera dedicar tiempo para abordar cuestiones de formación para la ciudadanía depende solo de su voluntad, nada lo obliga, no hay un lineamiento único de formación para la ciudadanía que alcance a todas las materias".

En un sentido similar a estas expresiones, otro directivo de centro educativo (Tacuarembó, abril de 2016) manifestó:

"Si bien se trata de enseñar a los alumnos la importancia que tiene su participación en las dinámicas propias del centro, en realidad sucede que las condiciones necesarias para que dicha participación acontezca no están presentes, como pasa con los tiempos institucionales que las actividades de participación estudiantil requieren. Cuando, por ejemplo, se eligen los Consejos de Participación los alumnos organizan las listas de votación y luego votan, pero cuando hay instancias de reuniones asisten los estudiantes que resultaron elegidos por sus pares pero no asisten los padres ni los representantes de la comunidad. Los alumnos junto con los docentes integrantes del Consejo arman proyectos que cuando son presentados para ser llevados a cabo hay miles de impedimentos interpuestos por parte de las mismas autoridades de los centros para lograr desarrollarlos (falta de recursos, falta de tiempo...), y así se termina el año y queda todo en la nada".

Asimismo, cabe citar lo que un docente entrevistado (profesor de Historia, Tacuarembó, abril de 2016) señaló: 
"Las acciones que conozco tendientes a formar en la ciudadanía en los liceos son sumamente puntuales o esporádicas. Ahora que me haces reflexionar sobre este tema por medio de las preguntas que me haces creo que faltan lineamientos que atraviesen toda la institución en este sentido; una especie de columna vertebral que sea educar para la ciudadanía. Quizá aún no existe porque no se lo ve como un tema importante".

También otros informantes calificados consultados (directivos y docentes) expresaron que las mismas sinergias que poseen los centros educativos del primer ciclo de Educación Secundaria en Uruguay inhiben el fomento de procesos que son clave en el desarrollo de una adecuada formación para la ciudadanía, procesos tales como la participación del estudiantado a nivel institucional, por ejemplo, en la presentación de propuestas de mejora del centro, en la formación de grupos de discusión para la toma de decisiones respecto a determinados aspectos de funcionamiento del centro educativo y en la organización de actividades de servicio a la comunidad donde se inserta el centro, entre otras acciones citadas. Esta debilidad fue atribuida, en todos los casos, a la inexistencia de lineamientos institucionales transversales que promuevan o favorezcan el adecuado desarrollo de propuestas concretas de formación para la ciudadanía.

Otro aspecto relevante para este estudio fue identificado durante el análisis documental, especialmente a partir del abordaje de los diferentes textos curriculares. Tal estudio reveló la exigua -o casi nula- transversalidad curricular que posee la formación para la ciudadanía en el Ciclo Básico. Es decir, pudo observarse que la amplia mayoría de los currículos del primer ciclo de Educación Secundaria no incorpora (ya sea en la parte de contenidos, objetivos, pautas de trabajo o evaluación) aspecto alguno que refiera de manera explícita al proceso de formación para la ciudadanía.

Esto implica que, en el Ciclo Básico, la falta de lineamientos transversales que se evidencian a nivel institucional debe ser entendida en el contexto de una falta de transversalidad en el ámbito estrictamente curricular y viceversa.

Es importante subrayar, entonces, un aspecto que en buena medida ayuda a explicar la situación antes descripta: el diseño curricular analizado en el caso uruguayo no posee ningún tipo de lineamiento transversal (Ilámese competencias básicas, objetivos generales, principios generales, criterios generales, entre otros nombres posibles) que permita y/o facilite incluir la formación para la ciudadanía (ni otras dimensiones formativas) en los programas de las distintas asignaturas.

De las entrevistas realizadas se obtuvieron algunas importantes observaciones referidas a la falta de transversalidad de la formación para la ciudadanía en los currículos analizados. Un directivo de centro educativo (Tacuarembó, mayo de 2016) expresó:

“Es difícil que un docente que no sea del área social sea consciente de que también, desde su asignatura, está formando para la ciudadanía, a través de las normas de comportamiento dentro del aula: saber escuchar, participar, respetar turnos, discutir ideas... Pero creo que la culpa de esto recae, principalmente, sobre los programas, que son absolutamente cerrados en sí mismos. Definitivamente la formación para la ciudadanía no es un eje transversal dentro de los programas, te diría que incluso no lo es siquiera para la totalidad de las materias sociales".

Por su parte, un docente entrevistado (profesor de Educación Social y Cívica, Tacuarembó, mayo de 2016) manifestó: 
“Hay ciertos procedimientos, como lectura y escritura, que creo ningún profesor siente que quedan por fuera de su clase, por lo tanto todos tratamos de colaborar en su tratamiento. Pero en el caso de la formación para la ciudadanía sé que no sucede igual. Por lo que habitualmente dialogamos en las salas de profesores con colegas de otras asignaturas la educación para la ciudadanía es vista como una cuestión de la cual debe ocuparse el docente de Educación Social y Cívica, entonces automáticamente los docentes de literatura, dibujo o ciencias físicas, por decir algunas materias, no lo ven como un tema abordable en sus clases".

Por último, cabe destacar que los cuatro directivos de centros educativos entrevistados coincidieron en que la falta de transversalidad que caracteriza a la formación para la ciudadanía tanto en el nivel institucional como en el estrictamente curricular debilita en medida importante los intentos de realizar trabajos sistemáticos y significativos en pro de tal formación. Un directivo en particular (Tacuarembó, abril de 2016) concluyó al respecto:

“En definitiva, de algún modo percibo como una doble carencia en este sentido: por un lado una carencia institucional porque es difícil, por ejemplo, llevar adelante procesos de participación del alumnado -me refiero a procesos genuinos, no a simulacros de participación-. Por otro lado, una carencia radicada en las aulas porque, como ya dije, no es un punto [la formación para la ciudadanía] que se enfoque de manera transversal".

En un sentido similar, otro directivo (Montevideo, junio de 2016) expresó:

“[...] la misma ausencia de un componente experiencial y transversal fuerte en los currículos ha conducido a obstaculizar la implementación de proyectos liceales que atiendan, por ejemplo, al mantenimiento de los espacios comunes. ¿Por qué? Porque [los alumnos] no los sienten como propios, como espacios colectivos, porque importa más lo mío, lo individual. Es difícil que el chiquilín sienta que el banco, por ejemplo, es suyo pero es de todos, que el salón es propio pero además es de todos. De esta forma se genera un dañino círculo vicioso: como los currículos no dejan mucho lugar a lo experiencial no se generan instancias significativas para que el alumno valore lo público [...]"

\section{Consideraciones finales}

Concluyendo, los contenidos de los textos abordados en los casos de Cataluña y Uruguay presentan acusadas diferencias en lo que respecta a cómo está planteada la formación para la ciudadanía.

En el caso de Cataluña las referencias a la transversalidad de tal formación en los niveles institucional y de aula son una constante. Asimismo, tales niveles son considerados por los informantes consultados de forma integrada en el propósito de formar para la ciudadanía.

En el caso de Uruguay los documentos analizados no expresan que la formación para la ciudadanía deba ser planteada de manera transversal en el nivel institucional ni en el de aula. Las entrevistas realizadas nos han permitido contextualizar esta carencia evidenciada y dimensionar algunas de las problemáticas que de ella derivan.

Estas circunstancias identificadas tanto en el caso catalán como en el uruguayo conviene aprehenderlas a la luz de lo que plantea Pedró (2003) respecto a los dos modelos curriculares que la investigación educativa comparada (especialmente la realizada en el ámbito europeo) ha identificado en lo que refiere a los procesos de formación para la ciudadanía en el ámbito de la educación formal. 
Por un lado se encuentra el modelo curricular que confiere a la educación ciudadana una situación ordinaria, con un estatus escolar bajo en relación a las restantes asignaturas (sea por la poca cantidad de horas semanales asignadas a la asignatura o porque la misma forma parte del diseño curricular de un solo curso o grado). Aquí estaría incluido el caso uruguayo. De todos modos cabe tener en cuenta que para Pedró (2003) la formalización de la educación ciudadana en una materia específica es, en sí misma, una cuestión ambivalente ya que, por una parte, en contextos escolares muy tradicionales la concreción de tal materia supone un paso importante en la valoración de la educación cívica que, por medio de tal formalización, se vuelve un contenido curricular relevante. Sin embargo, por otra parte, dicha formalización puede que sea también una estrategia inadecuada puesto que parece circunscribir la educación cívica a los lindes de un determinado programa de contenidos.

Por otro lado está el modelo curricular que asume a la educación ciudadana como una materia transversal. Aquí estaría incluido el caso catalán pero con el agregado de que en la Educación Secundaria Obligatoria también tienen cabida dos asignaturas específicas (Educación para la ciudadanía y los derechos humanos, en tercer grado, y Educación éticocívica, en cuarto grado). Esta situación que expone el caso catalán hace que la siguiente observación-problema que plantea Pedró (2003: 248) quede parcialmente resuelta:

“Considerarla una materia transversal tiene ventajas y, al mismo tiempo, inconvenientes. La principal ventaja es que, si realmente pasa a ser una cuestión prioritaria en el proyecto educativo del centro escolar, sería tanto como decir que la educación cívica está presente en todas y cada una de las actividades que se desarrollan en él, en todas las asignaturas pero también en la misma organización del centro. Y esta misma ventaja es también, paradójicamente, su principal inconveniente puesto que al ser transversal acostumbra a suceder que no cuenta con un responsable docente claro - cosa que no ocurriría si se tratara de una asignatura-, ni tampoco con una secuenciación específica de objetivos ni con su correspondiente programa de contenidos".

Según lo evidenciado y analizado en este estudio parece bastante patente que en Uruguay es necesario avanzar -y mucho- en lo que refiere a la configuración de lineamientos genuinos en materia de formación para la ciudadanía en el ciclo educativo considerado.

\section{Referencias bibliográficas}

Aiello de Almeida, M. (2010). La paz: camino para el cambio social. Buenos Aires: CEDSICiudad Nueva.

Aláez, B. (2005). Nacionalidad y ciudadanía: una aproximación histórico-funcional. Revista electrónica Historia Constitucional, № 6. Recuperado de https://dialnet.unirioja.es/descarga/ articulo/1254738.pdf

Álvarez, C. y San Fabián, J. L. (2012). La elección del estudio de caso en investigación educativa. Gazeta de Antropología, No 28/1. Recuperado de http://digibug.ugr. es/bitstream/handle/10481/20644/G28_14Carmen_Alvarez-JoseLuis_SanFabian. pdf? sequence $=11$ \&isAllowed $=y$

Anchustegui, E. (2011). Derechos humanos y modelos de ciudadanía. Revista electrónica de Filosofía y Psicología Límite, Vol. 6, No 24. Recuperado de http://www.redalyc.org/articulo. oa?id=83622474002 
Andrés, F. J. (2007). Ciudadanía romana y cosmopolitismo moderno. Revista Hispania antiqua, $\mathrm{N}^{\circ} 31,253-265$.

Audigier, F. (2000). Instruction civique, éducation civique, éducation à la citoyenneté, éducation aux citoyennetés... Changement du nom, changement de contenu? Vers une citoyenneté européenne. París: CANOPÉ, Académie de Dijon.

Berger, P. L. y Huntington, S. P. (2002). Globalizaciones múltiples. La diversidad cultural en el mundo contemporáneo. Barcelona: Paidós.

Buxarrais, M. (2009). Educación para la ciudadanía. ¿Qué tipo de ciudadanía? Revista Aula de Innovación Educativa, № 186, 38-42.

Cox, C. (2006). Jóvenes y ciudadanía política en América Latina. Desafíos al currículo. Revista PRELAC, N³, 64-73.

Fariñas, M. (1999). Los derechos humanos desde la perspectiva sociológico-jurídica a la actitud postmoderna. 2.a edición, Madrid: Dykinson.

González, E. (2011). Ciudadanía, identidades complejas y cultura política en los manuales escolares andaluces de educación para la ciudadanía y los derechos humanos. Tesis doctoral. Universidad de Granada. Recuperado de https://hera.ugr.es/tesisugr/19722813.pdf

Held, D. y McGrew, A. (2003). Globalización / Antiglobalización. Sobre la reconstrucción del orden mundial. Barcelona: Paidós.

Horrach, J. A. (2009). Sobre el concepto de ciudadanía: historia y modelos. Revista electrónica de Filosofía Factótum, № 6. Recuperado de http://www.revistafactotum.com/revista/f_6/ articulos/Factotum_6_1」A_Horrach.pdf

López de Cordero, M. (2011). La educación para la ciudadanía y derechos humanos: Una asignatura orientada a favorecer la convivencia. Tesis doctoral, Universidad de Barcelona. Recuperado de http://diposit.ub.edu/dspace/bitstream/2445/43115/4/01.MLdC TESIS.pdf

Meheut, M. (2000). Les valeurs qui fondent la citoyenneté européenne. Vers une citoyenneté européenne. París: CANOPÉ, Académie de Dijon.

Pagès, J. (2009). Competencia social y ciudadana. Revista Aula de Innovación Educativa, Nº $187,7-11$.

Pedró, F. (2003). ¿Dónde están las Ilaves? Investigación politológica y cambio pedagógico en la educación cívica. Aprendiendo a ser ciudadanos. Experiencias sociales y construcción de la ciudadanía entre los jóvenes. Madrid: Instituto de la Juventud. Ministerio de Trabajo y Asuntos Sociales.

Pérez, A. E. (2002). Ciudadanía y definiciones. Cuadernos Doxa: Cuadernos de Filosofía del Derecho, N 25. Recuperado de http://www.cervantesvirtual.com/obra/n-25---2002/

Red Eurydice. (2012). La educación para la ciudadanía en Europa. Recuperado de https:// sede.educacion.gob.es/publiventa/descarga.action?f_codigo_agc=16077_19 
Redon, S. (2010). La escuela como espacio de ciudadanía. Revista Estudios Pedagógicos, Vol. $36, N^{\circ} 2,213-239$.

Salazar, O. (2001). La ciudadanía compleja como fundamento de la Paz Social. Instituto de la Paz y Conflictos de la Universidad de Granada. Recuperado de http://ipaz.ugr.es/wpcontent/files/publicaciones/ColeccionEirene/eirene20/eirene20ap4.pdf

Stake, R. E. (1998). Investigación con estudio de caso. Madrid: Morata.

\section{(Endnotes)}

${ }^{1}$ En algunos currículos, como en el de Ciencias Sociales, Geografía e Historia, la Competencia social y ciudadana se encuentra incluida de forma -podríamos decir- natural y extendida, mientras que en otros, como en el de Matemáticas, esta inclusión se presenta redactada de forma mucho más exigua.

2 Esto es, que comprenda de forma compleja las dimensiones social, política, jurídica, ecológica y tecnológica, entre otras. 